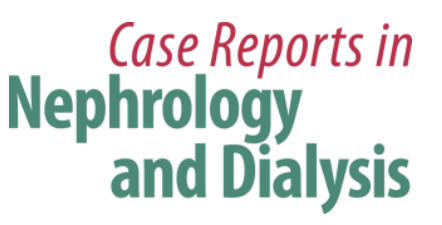

Case Rep Nephrol Dial 2016;6:1-7

\title{
Successful Treatment of Dual-Positive Anti-Myeloperoxidase and Anti-Glomerular Basement Membrane Antibody Vasculitis with Pulmonary-Renal Syndrome
}

\author{
Jinxian Huang Ling Wu Xiaoyan Huang Yan Xie Jinquan Yu \\ Jin Yang Huiqiong Fang Lijun Zhang
}

Rheumatology Department, The University of Hong Kong Shenzhen Hospital, Shenzhen, China

\section{Key Words}

Myeloperoxidase-antineutrophil cytoplasmic antibody · Anti-glomerular basement membrane antibody $\cdot$ Vasculitis $\cdot$ Pulmonary fibrosis $\cdot$ Mediastinal mass

\begin{abstract}
Antineutrophil cytoplasmic antibody (ANCA)-associated vasculitis and anti-glomerular basement membrane (GBM) disease are two separate diseases, while sometimes they can coexist together. The exact mechanisms are not clear, but due to the rapid progression and poor prognosis, prompt and aggressive treatment is usually required. We treated with steroids combined with cyclophosphamide and rituximab an 84-year-old man with ANCA-associated vasculitis and anti-GBM disease who had prior pulmonary fibrosis and a coexisting anterosuperior mediastinal mass. Conventional therapy including steroids, plasmapheresis and cyclophosphamide failed to attenuate the anti-GBM disease, yet he responded to an alternative treatment of rituximab. This case suggests the efficacy of steroids and immunosuppressant for the treatment of a dual-positive case with an anterosuperior mediastinal mass.
\end{abstract}




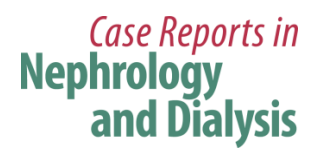

Case Rep Nephrol Dial 2016;6:1-7

DOI: $10.1159 / 000443163$

(C) 2016 The Author(s). Published by S. Karger AG, Basel www.karger.com/cnd

Huang et al.: Successful Treatment of Dual-Positive Anti-Myeloperoxidase and Anti-GBM Antibody Vasculitis with Pulmonary-Renal Syndrome

\section{Introduction}

Ever since 1989, dual-positive cases with anti-glomerular basement membrane (GBM) antibody and antineutrophil cytoplasmic antibody (ANCA) have been described in the literature and the implication of these antibodies has been discussed. According to the latest classification of vasculitis [1], ANCA-associated vasculitis (AAV) and anti-GBM disease both belong to the category of small-vessel vasculitis and might share some mechanisms in common. Although the exact role of dual positivity of ANCA and anti-GBM antibody remains to be clarified, there is important clinical evidence that ANCA may represent a serologic marker of good prognosis in anti-GBM disease. Patients have a poor prognosis when presenting with severe disease and initially behave more like having anti-GBM disease than vasculitis. Renal survival in dual-positive patients is usually not better than that in anti-GBM-positive patients and is worse compared with patients with myeloperoxidase (MPO)-ANCAs only.

Prior pulmonary fibrosis (PF) in dual anti-GBM antibody and ANCA cases was seldom reported [2-4] and its clinical significance and the association with disease is unclear. Lung involvement occurs presumably because of lung injury and the exposure of new epitopes to the immune system. The reported cases were all from an Asian population from Japan. The clinical course progressed fast and the prognosis was consistently very poor. The reasons for death included pulmonary hemorrhage, organ failure, infection and tumor.

Here, we present an experience of treatment of a dual anti-GBM-antibody- and ANCApositive vasculitis in a patient with preexisting PF and anterosuperior mediastinal mass. In our study, the case is evaluated clinically, serologically and pathologically.

\section{Case Report}

An 84-year-old male was admitted to our respiratory ward on November 23 last year because of intermittent fever with maximum temperature not exceeding 38.5 centigrade for more than 10 days, accompanied by cough with sputum and fatigue. He was a retired army pilot and a nonsmoker. He was treated with acetylcysteine due to PF detected by annual physical examination 5 years ago, with a coexistent anterosuperior mediastinal mass, which did not show much progression. He also had a past history of diabetes for 20 years.

On admission, his temperature was 37.6 centigrade. On physical examination, a fine moist rale over both lower lung fields was noted. Skin exam did not reveal any rash. Musculoskeletal exam revealed no joint swelling or tenderness and he had no edema of the limbs. Neurological exam was normal.

Complete blood count showed eosinophilia with an absolute value of eosinophils of 1.71 $\times 10^{9} / \mathrm{l}(13 \%)$ ). Renal function tests were normal (urea $4.5 \mathrm{mmol} / \mathrm{l}$, creatinine $97 \mu \mathrm{mol} / \mathrm{l}$ ) on admission. C-reactive protein was $47.7 \mathrm{mg} / \mathrm{l}$. Chest X-ray revealed thickened lung marking in both lower lung fields (fig. 1a). Empirical antibiotics treatment with amoxicillin potassium and doxycycline was used and was changed to piperacillin tazobactam and then tienam but the patient's symptom did not resolve. Blood culture, sputum culture, respiratory virus, sputum acid-fast bacillus smear, Streptococcus pneumoniae antigen, corps antigen, Mycoplasma pneumoniae DNA, tuberculosis DNA and tumor markers were all negative. Complement component C3 was $0.73 \mathrm{~g} / \mathrm{l}$ and immunoglobulin G (IgG) was $17.94 \mathrm{~g} / \mathrm{l}$. Antinuclear antibodies and anti-double-stranded DNA antibodies were negative. Indirect immunofluorescence revealed perinuclear ANCA positive with anti-MPO ANCA specificity by ELISA $(125 \mathrm{RU} / \mathrm{ml}$, normal $<20 \mathrm{RU} / \mathrm{ml}$ ); immunofluorescence for cytoplasmic ANCA, and ELISA for antiproteinase 3 ANCA were negative, but anti-GBM antibodies titer (ELISA) was positive at a titer of 
Huang et al.: Successful Treatment of Dual-Positive Anti-Myeloperoxidase and Anti-GBM Antibody Vasculitis with Pulmonary-Renal Syndrome

1:32. Tuberculosis screening for bronchoalveolar lavage fluid via bronchoscope was negative. Chest CT scan showed honeycombing, reticular and ground glass opacities in the lower lung fields (fig. 1b). Pulmonary function was roughly normal. Lung biopsy showed eosinophilia with vasculitis changes and granuloma formation (fig. 2). During his stay, the renal function was deteriorated (creatinine gradually increased to $171 \mu \mathrm{mol} / \mathrm{l}$ ). Percutaneous renal biopsy was performed and immunofluorescence microscopy disclosed linear staining of glomerular capillary basement membrane for IgG and C3, diagnostic for anti-GBM disease (fig. 3).

Diagnosis of AAV with coexistent anti-GBM disease in the patient with acute pulmonaryrenal syndrome and long-existing $\mathrm{PF} /$ anterosuperior mediastinal mass was made. The patient was then given a regimen consisting of i.v. methylprednisone $(80 \mathrm{mg} /$ day $)$ with i.v. cyclophosphamide $0.4 \mathrm{~g}$ every week and plasmapheresis (PE) every other week for 6 times. Perinuclear ANCA/anti-MPO ANCA returned to normal 1 week later but anti-GBM antibodies were still positive with the same titer. The symptoms disappeared without relapse. Steroids were then tapered to $60 \mathrm{mg} /$ day. Unfortunately, the patient experienced shortness of breath, palpitation and hypotension during PE, ongoing continuous bleeding from the puncture point of the femoral vein with a dramatic drop of platelet count $\left(32 \times 10^{9} / \mathrm{l}\right.$ at the lowest), which suggested the diagnosis of heparin-induced thrombocytopenia (HIT). Bilateral lower extremity compression ultrasonography was applied in this patient to rule out silent deep vein thrombosis. PE was discontinued and rivaroxaban was used until the platelet count returned to normal. Anti-GBM antibody was still positive and renal function did not ameliorate after 3 weeks of cyclophosphamide. Therefore, immunosuppressant was switched to rituximab $700 \mathrm{mg} /$ week. Anti-GBM antibody turned to negative after 1 month and creatinine decreased to $149 \mu \mathrm{mol} / \mathrm{l}$. Mycophenolate mofetil with the dosage of $1.5 \mathrm{~g} /$ day was then used for maintenance treatment and oral prednisone gradually decreased to $25 \mathrm{mg} /$ day.

At the latest follow-up visit after around 4 and a half months' treatment, all the antibodies remained negative and creatinine was $123 \mu \mathrm{mol} / \mathrm{l}$. Repeated chest CT revealed a novel nodule in the upper lobe of the left lung $(0.9 \times 1.0 \mathrm{~cm})$ with concomitant mild progression of interstitial changes. A further PET/CT showed the novel lesion with a standardized uptake value of 3.0 suggesting inflammatory pseudotumor. Meanwhile, the anterosuperior mediastinal mass was remarkably diminished without thymectomy intervention (fig. 4).

\section{Discussion}

The case in our study had previously demonstrated PF but tests for anti-GBM antibody and ANCA were not performed at those times. We are unable to figure out whether ANCA or anti-GBM were previously positive. It was postulated that ANCA-associated glomerular or pulmonary damage releases, exposes, or possibly alters basement membrane material, rendering it autoimmunogenic and the anti-GBM antibodies pathogenic and probably reacted with the NC1 domain of the $\alpha 3$ chains of type IV collagen. Although the hypothesis that endogenous basement membrane components induce anti-GBM antibodies in patients with ANCA is plausible, there is no direct evidence to support it.

$\mathrm{PF}$ is a rare but clinically relevant manifestation occurring in association with AAV. In a survey of a series of 49 patients with PF associated with AAV, the eosinophil level was a poor prognostic factor, while initial pattern on thoracic high-resolution CT was not a prognostic factor [5]. The presence of positive MPO-ANCA was reported to be an unfavorable prognostic factor in patients with PF [6], and the detection of antibodies is necessary in monitoring dis- 


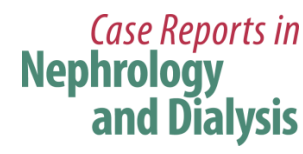

\begin{tabular}{l|l}
\hline Case Rep Nephrol Dial 2016;6:1-7 \\
\hline DOI: 10.1159/000443163 & $\begin{array}{l}\text { C 2016 The Author(s). Published by S. Karger AG, Basel } \\
\text { www.karger.com/cnd }\end{array}$ \\
\hline
\end{tabular}

Huang et al.: Successful Treatment of Dual-Positive Anti-Myeloperoxidase and Anti-GBM Antibody Vasculitis with Pulmonary-Renal Syndrome

ease relapse [7]. Our case adds knowledge to the former dual-positive cases with prior PF, in which the clinical presentation can also have a slower and better outcome.

Concerning eosinophilia in white blood cell and lung tissue, the diagnosis of eosinophilic granulomatosis with polyangiitis (EGPA) was suspected. EGPA was formerly called ChurgStrauss syndrome. This case did not have any asthma history and neither known mononeuropathy nor polyneuropathy nor paranasal sinus abnormality was discovered. Moreover, roentgenography did not show any nonfixed pulmonary infiltrates. Thus, the criteria of Churg-Strauss syndrome were not met for this case.

Rituximab has been used in AAV since 2003 with favorable efficacy [8]. It was also reported to be used in refractory anti-GBM disease in case series [9]. The reason for the application of rituximab as alternative treatment for this case was irreversible renal dysfunction and persistent positive anti-GBM antibody suggestive of unfavorable outcome. We changed immunosuppressant to mycophenolate mofetil after remission induction while regarding the high incidence of infection complication. The whole response of this case to the treatment was satisfactory and lived up to our anticipation.

To our knowledge, this is the first case report of the coexistence of an anterosuperior mediastinal mass in a dual-positive case. Without the evidence of pathology, we were not able to confirm the diagnosis and histological classification of the mediastinal mass. However, according to the location, character and border of the mass as shown on CT scan, as well as suggestive benign mass and implicated origin from the thymus as shown on PET/CT, the diagnosis of thymoma was most suspected. Thymoma has been associated with a wide range of autoimmune disorders. Thymectomy did not always induce clinical and immunologic remissions of associated autoimmune syndromes and, in some cases, caused disease progression or switch from 1 autoimmune disease to another [10]. In consideration of venerable age with moderate basic diseases as relative contraindication for operation in our case, we did not choose thymectomy as the first-line treatment for the mediastinal mass. Beyond our expectation, we happened to discover a dramatic diminishment of the anterosuperior mediastinal mass size after less than half a year.

The patient experienced dramatic thrombocytopenia during plasma exchange. Concerning prior heparin exposure, platelet count drop and hemorrhage at local site from the femoral vein, the diagnosis of HIT was made. Fortunately, thrombosis did not form. HIT is reported to occur in $0.2-5 \%$ of heparin-treated adults. In a historical series of 62 untreated patients with isolated HIT, the cumulative incidence of thromboembolism at 30 days was $53 \%$. Most events occurred within 10 days of heparin discontinuation, a period corresponding to platelet recovery. The optimal duration of anticoagulation in persons with HIT without thrombosis is unknown. We therefore started anticoagulation in this case until the platelet count had recovered to a stable plateau at a minimum, and we considered extending therapy to a month after heparin cessation due to the presence of additional thrombotic risk factors including vasculitis [11], diabetes and elder age. Further study is essential to understand if the association of anti-GBM disease and renal vein thrombosis as seen in the reported case was pure coincidence or in fact occurs more frequently [11].

As the conventional anticoagulants bear a considerable bleeding risk, new anticoagulants provide new treatment options [12]. In theory, new oral inhibitors of thrombin and FXa constitute rational therapies for HIT and also offer the promise of simplifying management. Currently, the direct-acting oral anticoagulants are FDA approved for venous thromboembolism, yet have limited evidence in both in vitro and clinical HIT studies. It is unlikely that a large randomized controlled trial will be performed because of the low prevalence of HIT.

Currently, rivaroxaban is the most studied of these agents. It did not cause platelet activation or aggregation in the presence of HIT antibodies. Additionally, rivaroxaban did not 
interact with PF4, nor did it trigger the release of PF4 from platelets [13-15]. Concerning its relatively extensive use, safety and efficacy, rivaroxaban was chosen as prevention of thrombosis in our case and off-label drug use was approved by our hospital's pharmacological department. This might be a good beginning for future application of prophylaxis treatment for emergency situations like HIT.

\section{Conclusion}

Rituximab may represent an additional and/or alternative therapy in the induction treatment of concomitant AAV and anti-GBM disease.

\section{Statement of Ethics}

The authors have no ethical conflicts to disclose.

\section{Disclosure Statement}

The authors declare that they have no conflict of interest.

\section{References}

1 Jennette JC, Falk RJ, Bacon PA, et al: 2012 revised International Chapel Hill Consensus Conference Nomenclature of Vasculitides. Arthritis Rheum 2013;65:1-11.

$\longrightarrow 2$ Kobayashi M, Saito M, Minoshima S, et al: A case of progressive systemic sclerosis with crescentic glomerulonephritis associated with myeloperoxidase-antineutrophil cytoplasmic antibody (MPO-ANCA) and anti-glomerular basement antibody (anti-GBM Ab). Jpn J Nephrol 1995;37:207-211.

-3 Yamazaki K, Kanehira K, Inaba Y, et al: A case of rapidly progressive glomerulonephritis with antiglomerular basement membrane antibody in the course of MPO-ANCA positive interstitial pneumonia. Nihon Jinzo Gakkai Shi 2012;54:1203-1208.

4 Bal A, Das A, Gupta D, et al: Goodpasture's syndrome and p-ANCA associated vasculitis in a patient of silicosiderosis: an unusual association. Case Rep Pulmonol 2014;2014:398238.

$\checkmark 5$ Comarmond C, Crestani B, Tazi A, et al: Pulmonary fibrosis in antineutrophil cytoplasmic antibodies (ANCA)-associated vasculitis: a series of 49 patients and review of the literature. Medicine (Baltimore) 2014;93:340-349.

6 Homma S, Matsushita H, Nakata K: Pulmonary fibrosis in myeloperoxidase antineutrophil cytoplasmic antibody-associated vasculitides. Respirology 2004;9:190-196.

7 Sinclair D, Stevens JM: Role of antineutrophil cytoplasmic antibodies and glomerular basement membrane antibodies in the diagnosis and monitoring of systemic vasculitides. Ann Clin Biochem 2007;44:432-442.

-8 McGregor JG, Hogan SL, Kotzen ES, et al: Rituximab as an immunosuppressant in antineutrophil cytoplasmic antibody-associated vasculitis. Nephrol Dial Transplant 2015;30(suppl 1):i123-i131.

-9 Arzoo K, Sadeghi S, Liebman HA: Treatment of refractory antibody mediated autoimmune disorders with an anti-CD20 monoclonal antibody (rituximab). Ann Rheum Dis 2002;61:922-924.

$\rightarrow 10$ Parambil JG, Keogh KA, Fervenza FC, et al: Microscopic polyangiitis associated with thymoma, exacerbating after thymectomy. Am J Kidney Dis 2006;48:827-831.

11 Emmi G, Silvestri E, Squatrito D, et al: Thrombosis in vasculitis: from pathogenesis to treatment. Thromb J 2015;13:15.

12 Miyares MA, Davis KA: Direct-acting oral anticoagulants as emerging treatment options for heparin-induced thrombocytopenia. Ann Pharmacother 2015;49:735-739.

13 Hantson P, Lambert C, Hermans C: Rivaroxaban for arterial thrombosis related to heparin-induced thrombocytopenia. Blood Coagul Fibrinolysis 2015;26:205-206.

14 Ng HJ, Than H, Teo EC: First experiences with the use of rivaroxaban in the treatment of heparin-induced thrombocytopenia. Thromb Res 2015;135:205-207. 
Case Reports in

Nephrology

and Dialysis

\begin{tabular}{l|l}
\hline Case Rep Nephrol Dial 2016;6:1-7 \\
\hline DOI: 10.1159/000443163 & $\begin{array}{l}\text { ○ 2016 The Author(s). Published by S. Karger AG, Basel } \\
\text { www.karger.com/cnd }\end{array}$ \\
\hline
\end{tabular}

Huang et al.: Successful Treatment of Dual-Positive Anti-Myeloperoxidase and Anti-GBM Antibody Vasculitis with Pulmonary-Renal Syndrome

15 Linkins LA, Warkentin TE: Design of the rivaroxaban for heparin-induced thrombocytopenia study. J Thromb Thrombolysis 2014;38:485-492.
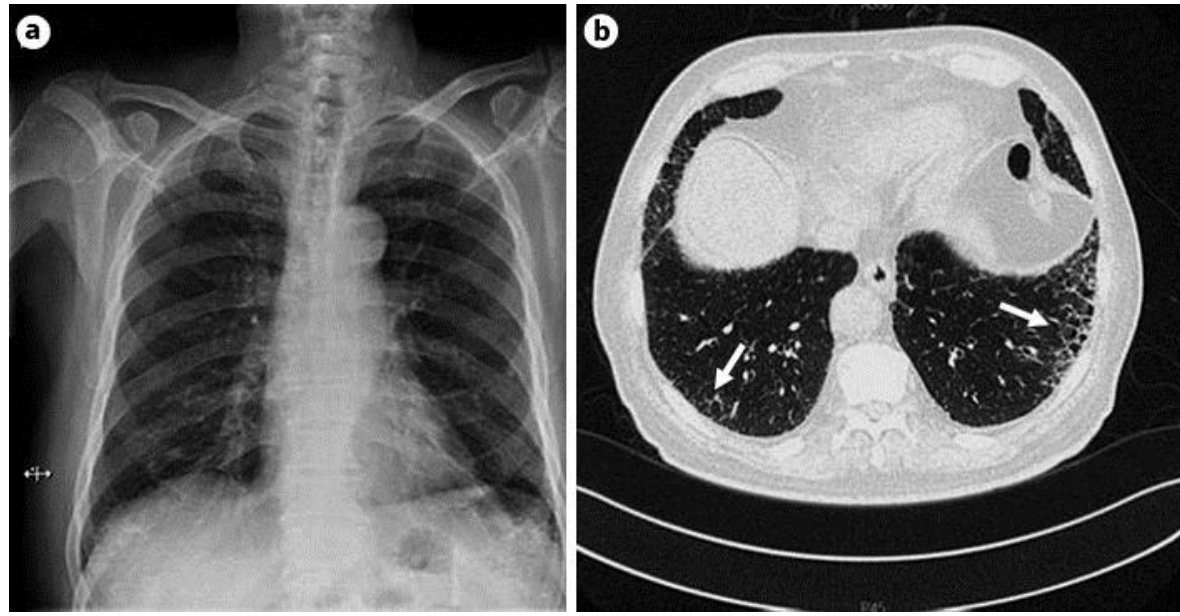

Fig. 1. Chest X-ray (a) and CT scan (b) on admission. a Chest X-ray revealed thickened lung marking in both lower lung fields. $\mathbf{b}$ Chest CT scan showed honeycombing, reticular and ground glass opacities in the lower lung fields (arrows).

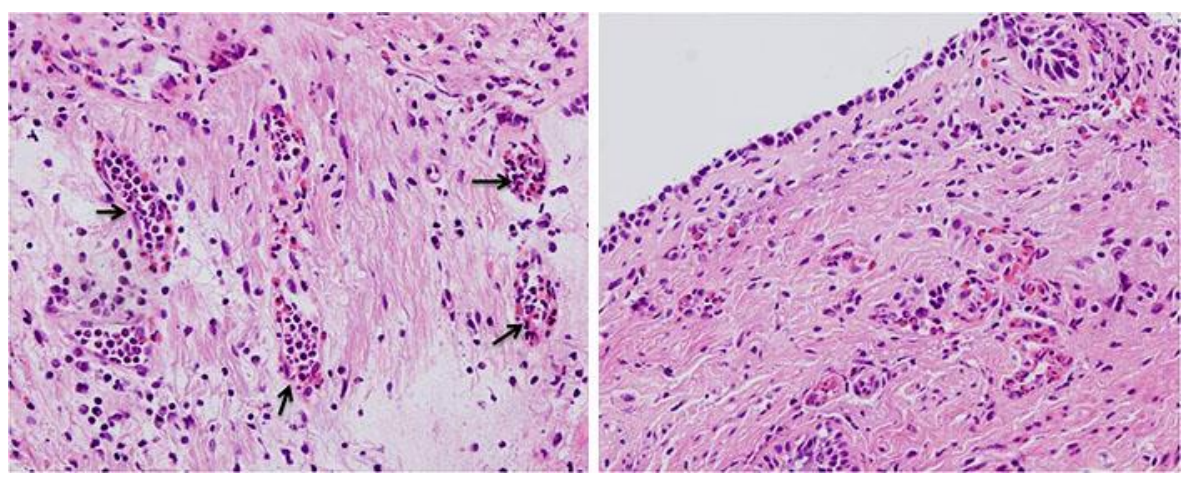

Fig. 2. Lung biopsy showed eosinophilia (arrows), lymphocyte and plasma cell infiltration and multiple eosinophil inflammation without granuloma formation. 


\section{Case Reports in \\ Nephrology \\ and Dialysis}

\begin{tabular}{l|l}
\hline Case Rep Nephrol Dial 2016;6:1-7 \\
\hline DOI: 10.1159/000443163 & $\begin{array}{l}\text { ○ } 2016 \text { The Author(s). Published by S. Karger AG, Basel } \\
\text { www.karger.com/cnd }\end{array}$ \\
\hline
\end{tabular}

Huang et al.: Successful Treatment of Dual-Positive Anti-Myeloperoxidase and Anti-GBM Antibody Vasculitis with Pulmonary-Renal Syndrome

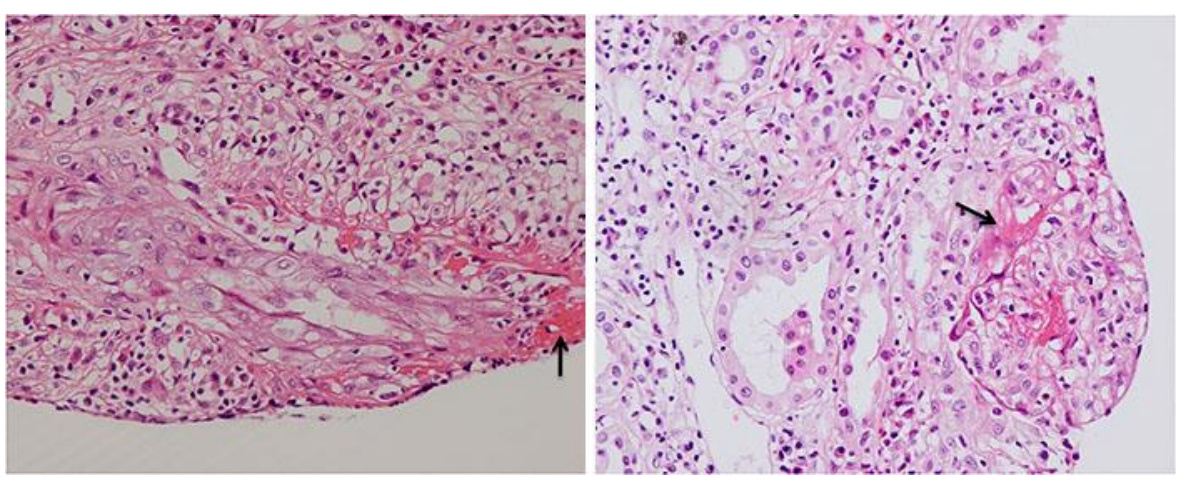

Fig. 3. Light microscopy and immunofluorescent findings on renal biopsy. The biopsy specimen contained 6 glomeruli, 2 of which with gomerulosclerosis. Another 2 showed segmental necrosis (arrows). Two granuloma-like structures were seen in situ. Diffuse chronic inflammatory cell infiltration with predominant lymphocytes and plasma cells was observed in mesenchyma.
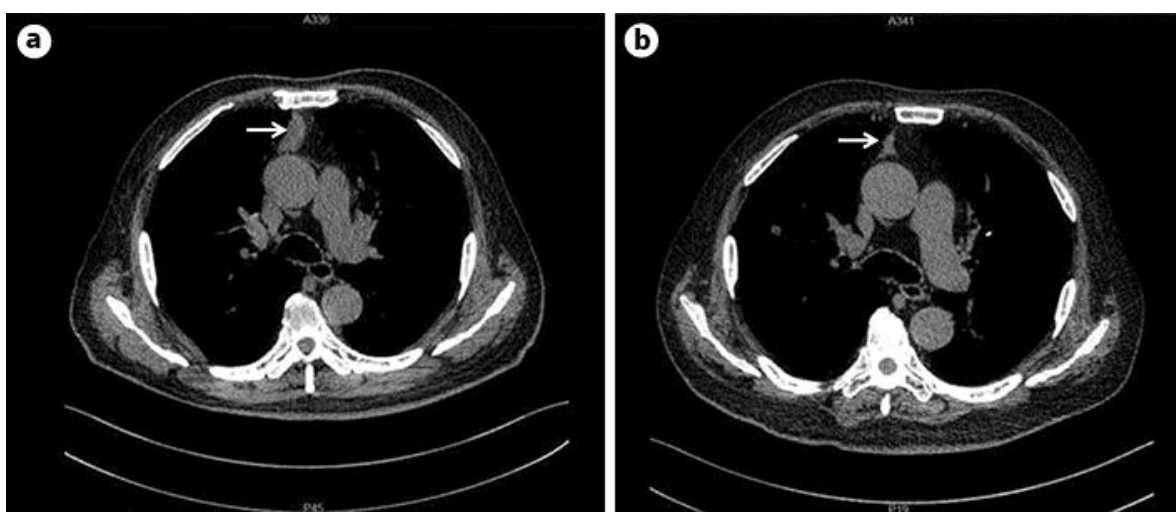

Fig. 4. Chest CT scan showing anterosuperior mediastinal mass before (a; arrow) and after (b; arrow) treatment. a The size of the anterosuperior mediastinal mass was $3.5 \times 1.9 \mathrm{~cm}$ with CT $5 \mathrm{HU}$. $\mathbf{b}$ The same mass was diminished to $1.2 \times 1.0 \mathrm{~cm}$ with CT $16 \mathrm{HU}$. 